\title{
AprilCal: Assisted and repeatable camera calibration
}

\author{
Andrew Richardson Johannes Strom Edwin Olson
}

\begin{abstract}
Reliable and accurate camera calibration usually requires an expert intuition to reliably constrain all of the parameters in the camera model. Existing toolboxes ask users to capture images of a calibration target in positions of their choosing, after which the maximum-likelihood calibration is computed using all images in a batch optimization. We introduce a new interactive methodology that uses the current calibration state to suggest the position of the target in the next image and to verify that the final model parameters meet the accuracy requirements specified by the user.

Suggesting target positions relies on the ability to score candidate suggestions and their effect on the calibration. We describe two methods for scoring target positions: one that computes the stability of the focal length estimates for initializing the calibration, and another that subsequently quantifies the model uncertainty in pixel space.

We demonstrate that our resulting system, AprilCal, consistently yields more accurate camera calibrations than standard tools using results from a set of human trials. We also demonstrate that our approach is applicable for a variety of lenses.
\end{abstract}

\section{INTRODUCTION}

Applications such as visual odometry [14], dense reconstruction [8], [15], and colored point cloud segmentation [20] are fundamentally dependent on accurate calibrations in order to extra metrical data from images. The MATLAB and OpenCV packages are two popular systems for calibrating lenses [3], [4]. However, they can be error prone, especially for lenses with significant distortion. This stems from the fact that the quality of a calibration is dramatically affected by the user's choice of calibration images. A user who chooses poor calibration target positions may find the resulting model generalizes poorly to unseen examples. This challenge is particularly acute for novice users, who are not aware of the properties of the underlying estimation and optimization methods, or end-users in dramatically different fields [2]. Even experts may be unsure that the positions they have chosen will yield a sufficiently accurate calibration, as the number of images needed is not constant across lenses and should vary with the quality of the constraints. Consequently, standard practice is to collect many more images than necessary and verify that the model parameter uncertainty and training error are low; if the results are unsatisfactory, the calibration is repeated or updated with additional images. This process is unreliable, and not very satisfying from a theoretical standpoint.

Therefore, the primary goal of this work is to increase calibration repeatability and accuracy in a more principled

The authors are with the Computer Science and Engineering Department, University of Michigan, Ann Arbor, MI 48104, USA \{chardson, jhstrom, ebolson\}@umich.edu http://april.eecs.umich.edu

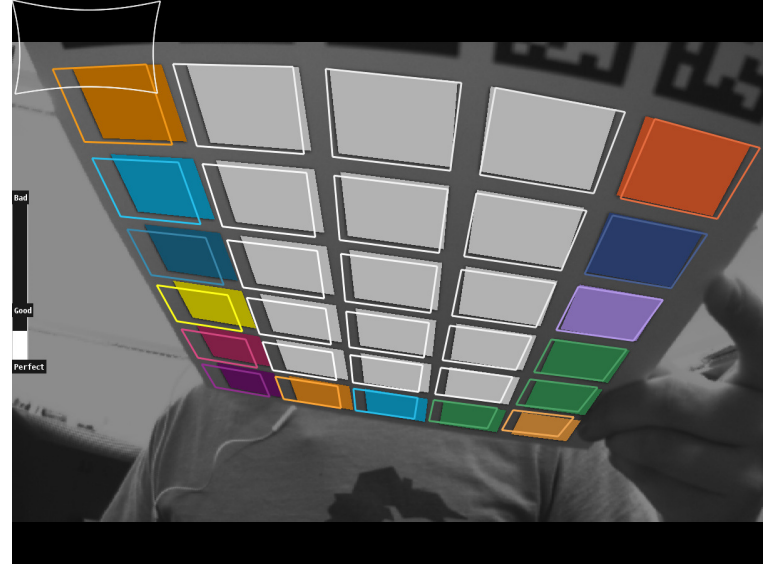

Fig. 1: The AprilCal GUI. Our system combines the ability to reason about unseen targets and a novel quality metric to make suggestions to the user about where to place the target. The user is notified that calibration is complete once the desired accuracy has been reached, typically achieving $<1$ pixel of error after 6-8 images.

fashion. We introduce a paradigm where fit quality is explicitly considered at each stage during a live calibration process. Specifically, we automatically consider many unseen target positions and suggest positions that will best improve the quality of the calibration. This is achieved using a novel quality metric based on the uncertainty of the calibration as measured in pixels. Previous toolboxes report the uncertainty of the model parameters, but the effect of these parameter uncertainties on pixel coordinates can be complex. We argue that worst-case uncertainty in pixels is more relevant for application performance and more natural for the user. Worstcase pixel uncertainty also serves as a principled basis to automatically determine when enough images have been collected.

We also introduce a new method for robustly bootstrapping a calibration that enables our system to make sensible recommendations even when little or no prior information is available about the lens. Our system also makes use of a calibration target composed of AprilTags [16], which, unlike previous approaches, can still be detected when individual markers are occluded. This enables a wider variety of target positions, which our method successfully exploits when making suggestions to the user.

We validated our camera calibration toolbox via a 16participant study mostly compromised of users who had never calibrated a camera. Despite their lack of expertise, they were consistently able to use our software to produce 
accurate calibrations. The same novice users also used the OpenCV calibration toolbox and invariably produced poorer calibrations, in some cases yielding errors of tens of pixels. In addition, we show that our toolbox can calibrate a wide range of lenses with an explicit accuracy guarantee.

The contributions of this work include:

- A framework for generating target position suggestions to guide users during camera calibration

- A new evaluation metric for camera calibration that enables high confidence in calibration parameters everywhere in the image

- A bootstrapping setup to enable interactive calibration even before all parameters are fully constrained

- An evaluation that demonstrates the robustness of our approach using a human-subjects study of 16 people, mostly novices.

AprilCal is released as part of the APRIL Robotics Toolkit and is available at http://april.eecs.umich.edu

\section{BACKGROUND}

A wide variety of camera calibration approaches exist, spanning different optimization methods, calibration target styles and intrinsic model designs. Many previous methods have used multiple views of a planar target [22], [3], [4] or a single view of a carefully constructed 3D target [13]. Other methods have used laser pointers or other bright lights to facilitate calibration of networks of cameras. Such approaches typically still require bootstrapping by calibrating some cameras in the network with a constructed target [1], [21]. All of these prior methods share the same approach to calibration: a user first collects a set of images, then runs a batch calibration process on that data. This is in contrast to our approach, where the entire calibration process is interactive and additional data is solicited until the desired accuracy has been achieved.

A dominant paradigm for calibration involves capturing several images of a planar target. These approaches (ours among them) make associations between points detected in the image and corresponding world points on the target whose relative position are known by construction [22]. Simultaneous optimization over the intrinsic parameters for the camera model and the extrinsics for each target yield an estimate of the model parameters. Using such an approach requires the choice of 1 ) optimization method and 2) lens model.

Among the many possible optimization techniques, we adopt a standard, iterative non-linear-least-squares approach, using a sparse matrix solver as the back-end. This method is roughly analogous to standard approaches in GraphSLAM and bundle adjustment [5], [12], [11]. Our calibration vector $x$ consists of all the model parameters (roughly 10) for the camera, in addition to the 6-DOF position of each calibration target. For each image containing $k$ extracted 2D image points, we add $2 k$ linearized constraints as rows in the Jacobian matrix $J$. Each row-pair corresponds to projecting a feature from a known 3D coordinate on the calibration target into pixel coordinates, capturing both the unknown position of the camera and the unknown camera parameters. Iterative solutions to Eqn 1 yield a locally-optimal set of model parameters for $x$.

$$
\begin{aligned}
J^{T} \Sigma_{z}^{-1} J \Delta x & =J^{T} \Sigma_{z}^{-1} r \\
x_{i+1} & =x_{i}+\Delta x
\end{aligned}
$$

Here, $\Sigma_{z}$ is the matrix of prior covariances for the target detector, and $r$ is the residual, the observed minus the predicted pixel coordinates for each point. The correct convergence of $x$ to the global minimum is sensitive to initialization of $x_{0}$; we will discuss our approaches to this in Section III-A.

There are also a wide variety of models for camera intrinsics, starting with the fundamental pinhole model [7]. However, using the ideal pinhole model in isolation will poorly capture the dynamics of most real world lenses, especially those with a wide field of view. Therefore, many models extend this method by accounting for the lens distortion explicitly. For example, the MATLAB toolbox uses a polynomial Taylor series with 3-5 distortion terms to approximate these effects after projecting with the pinhole camera model [3]. In contrast, we have found that a polynomial as a function of $\theta$, the angle from the principle axis, yields as good or better ${ }^{1}$ calibrations, often with fewer distortion terms for the lenses tested, increases the stability of the calibration process, and handles $Z \leq 0$. This is a reduced version of the model by Kannala and Brandt [10], which also includes tangential distortion.

The details of this angular polynomial model are shown in Equations 3-8, where $X, Y$, and $Z$ represent the 3D position of a point, $\theta$ the angle from the principal axis, $\psi$ the angle around the principal axis, $\boldsymbol{x}_{\boldsymbol{d n}}$ the distorted point before converting to distorted pixel coordinates, $\boldsymbol{x}_{\boldsymbol{d}}$, via the matrix $K$. The number of distortion coefficients is variable, though we use three to four in this work.

$$
\begin{aligned}
\theta & =\arctan 2\left(\sqrt{X^{2}+Y^{2}}, Z\right) \\
\psi & =\arctan 2(Y, X) \\
r(\theta) & =\theta+k_{1} \theta^{3}+k_{2} \theta^{5}+k_{3} \theta^{7}+k_{4} \theta^{9}+\ldots \\
\boldsymbol{x}_{\boldsymbol{d n}} & =[r(\theta) \cos (\psi), r(\theta) \sin (\psi)]^{T} \\
K & =\left[\begin{array}{ccc}
\boldsymbol{f}_{\boldsymbol{x}} & 0 & \boldsymbol{c}_{\boldsymbol{x}} \\
0 & \boldsymbol{f}_{\boldsymbol{y}} & \boldsymbol{c}_{\boldsymbol{y}} \\
0 & 0 & 1
\end{array}\right] \\
\boldsymbol{x}_{\boldsymbol{d} \boldsymbol{p}} & =K \cdot\left[x_{d n}, y_{d n}, 1\right]^{T}
\end{aligned}
$$

In a similar spirit to this paper, several others have sought to make calibration easier, less time consuming and less error prone. For example, the ROS calibration package for the PR2 now has specific guidelines for the user about which checkerboard positions are required for getting a "good" calibration [18]. However, even with good rules of thumb, it is still possible that a user will collect "bad" frames that

\footnotetext{
${ }^{1}$ The details of this analysis are omitted due to space limitations. However, AprilCal can perform model selection to evaluate all available models as a post-processing step. See http://april.eecs.umich.edu for details.
} 
lead to an inaccurate calibration. While it is possible to manipulate a calibration target automatically using a robotic arm [17], our approach can be used to choose desired target views during calibration. Others have shown that in some cases, it can be possible to calibrate a distorted camera using only a single image [2]. However, this approach does not explicitly constrain the accuracy of the resulting calibration, and works with only a very specific distortion model. In contrast, our method ensures that the user takes sufficient images to meet their desired level of accuracy, typically 6-8 images to achieve a $<1$ pixel confidence.

Finally, as suggested in Ranganathan's work on nonparametric intrinsics models [19], we adopt the use of a strict testing set to provide a more rigorous evaluation of the actual accuracy of the proposed method. This is in contrast to the standard practice of reporting only the training error.

\section{PROPOSED Method}

As outlined in Section I, our proposed method improves on the state of the art by offering a virtual calibration assistant that provides suggestions to users and automatically notifies them when the calibration has reached the specified accuracy. Our approach leverages a calibration target consisting of a mosaic of AprilTags [16], which can be detected robustly on the live video stream, even if parts of the target are occluded. Users interact with a GUI to match target positions suggested by our software. After each target position is achieved, the next best position is computed, repeating until the desired accuracy is achieved.

Our system divides the calibration into two sequential phases: bootstrapping, and uncertainty reduction. In the bootstrapping phase, we start with a restricted camera model with very few parameters, relaxing to the full model as new images of the target are added. Once all model parameters are fully constrained (typically after 3 images), we switch to the uncertainty reduction phase until the desired accuracy is achieved. Transition from the first to second phase is transparent: in both cases, the UI remains the same.

\section{A. Bootstrapping a live calibration from scratch}

Suggesting target positions is an inherently cyclic process: making good proposals that are actually realizable given the lens distortion requires a reasonably accurate calibration. However, a good calibration relies on having already captured several target positions to properly constrain all model parameters [22]. Therefore, to initialize the model parameters as quickly as possible, we initially use a reduced camera model; at the start of each calibration we assume that the focal center is at the center of the image and that there is no image distortion. This allows us to estimate the focal length after a single frame.

We can use this limited model immediately to choose the next-best target position, relaxing the reduced model to the full model, shown in Eqns. 3 - 8, as more frames are taken. This method is intended to select the target positions that best constrain the calibration while avoiding degenerate combinations [22]. We compute the calibration initialization using a standard intrinsics matrix estimation technique - estimating the Image of the Absolute Conic (IAC) from perpendicular vanishing points and then decomposing it to estimate the intrinsics matrix [22], [9]. Using this initialization method, we score potential suggestions by sampling from the observation model to empirically compute the uncertainty of the intrinsics estimate. In other words, we prefer suggestions that yield intrinsics estimates with low variance. In our implementation, we estimate the focal length over 20 trials, each time adding uniformly-random, zeromean noise to the image coordinates of the tag detections.

Some lenses generate too much distortion for IAC intrinsics matrix estimation. However, methods exist to remove the distortion from a single image [6], [2]. Such methods could be easily added to AprilCal, but this was unnecessary for the moderately distorted, wide field of view lenses tested in this work (see Figure 9).

Before the first suggestion can be shown to the user, we must obtain a cursory estimate of the camera calibration. We achieve this by automatically selecting the first image "behind the scenes" as the user moves the target to the center of the screen. However, the very first frame may not provide a robust initialization. To make this initialization robust, we score the live image stream and replace the first frame (removing the previous one) every time we find a new frame with a lower intrinsics uncertainty, either until a threshold is met, or the first suggestion has been computed and captured. This method reliably picks a satisfactory first frame because the user is guided to move the calibration target to a suggestion as soon as any frame has been captured and the intrinsics matrix has been estimated.

Once the calibration has been initialized, we can consider the effect of observing an unobserved frame on the uncertainty of the parameter estimates. For each candidate target position drawn from a coarse grid in pose space, we score the intrinsics estimate resulting from the combination of 1) the frames acquired so far (ignoring initial frames that were replaced) and 2) the projection of the candidate calibration target with the current estimates of the calibration parameters. As before, we sample from the observation model to estimate the uncertainty of the intrinsic parameters, choosing the suggestion that reduces the parameter variance the most.

In addition to providing full-rank constraints for all parameters of the complete camera model, the bootstrapping process also provides a good initialization for $x_{0}$ in the optimization described in Eqn.1. As the model is successively relaxed, we pass through the initialization from the previous step, yielding good estimates for all the intrinsics parameters. Once the distortion parameters are introduced, we initialize them to zero. Given sane intrinsics estimates, these parameters converge well in practice.

\section{B. Pixel-based calibration error metric}

Once all intrinsic parameters are fully constrained, the next goal is to find enough additional target observations so that we are confident that the resulting model parameters are 

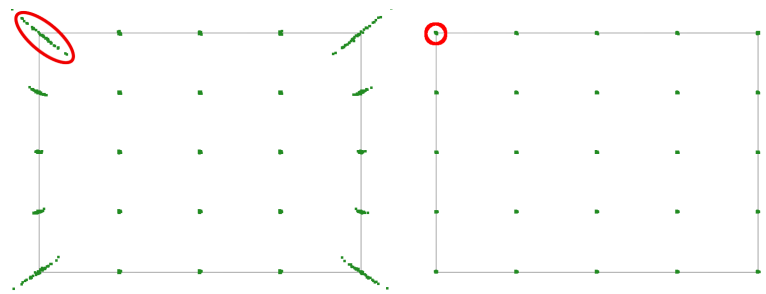

Fig. 2: Computing the Max Expected Reprojection Error (Max ERE) from the test points, and resulting error distributions after 3 and 5 images. Our sampling-based error metric can be used with any intrinsics model and allows us to automatically ensure the calibration is well constrained everywhere without requiring the user to collect a test set. The set of samples with the Max ERE are circled in red.

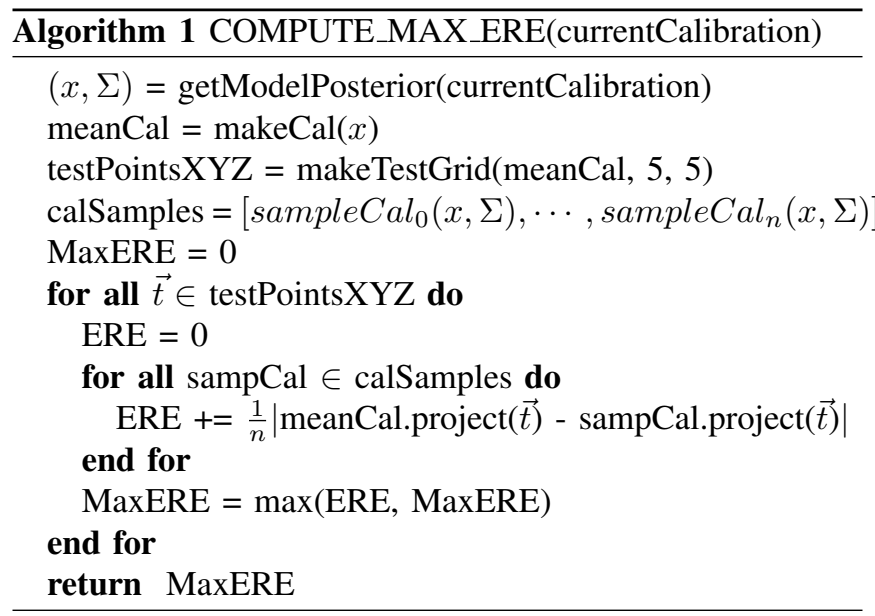

accurate. Previous approaches have used Mean Reprojection Error (MRE) and Mean Squared Error (MSE) as primary indicators of calibration quality. However, this is problematic because these are training errors, rather than testing errors. Using one of the prior works, if the training images are selected poorly, the resulting MRE could be low, yet the generalization performance (e.g. as measured on a test set) could be very poor. Unfortunately, collecting a proper testing set can be onerous - for our evaluation we use an expertselected set of 60 or more images from all over the camera's field of view. Especially for novice users, it is not reasonable to expect they would be able to collect a "good" testing set. Even for expert users, this process is time-consuming and requires careful attention.

Therefore, our approach is to derive a more principled estimate of the testing error that can be computed automatically given an intermediate state of the calibration. Our proposal is called "Max Expected Reprojection Error" (Max ERE), which we can compute at any stage during the calibration by sampling from the current posterior distribution over the model parameters. We then project a series of 3D points through each sampled calibration, producing a distribution of pixels for each test point, whose mean error is the "ERE". Finally, we take the max of the EREs over all the test points. This ensures that we will properly weight the part of the image where the model is currently the most uncertain. We choose the fixed 3D points carefully so that they will project into all parts of the image. Our current implementation uses a 5 x 5 grid of test points distributed so their projections will uniformly cover the image (see Figure 2). See Alg. 1 for an overview of the implementation.

Computation of the Max ERE uses the estimate of the marginal posterior covariance of the model parameters: $\bar{P}\left(m \mid z_{0}, \cdots, z_{n}\right)$. This distribution is derived by first computing the joint distribution of the model parameters and each target extrinsics, given all the observations of those targets. Suppose we have collected $n$ images of targets, then we can "marginalize-out" the target extrinsics:

$$
\bar{P}\left(m \mid z_{0}, \cdots z_{n}\right)=\int_{T_{0}, \cdots, T_{1}} P\left(m, T_{0}, \cdots T_{n} \mid z_{0}, \cdots z_{n}\right)
$$

where $m=\left\{f_{x}, f_{y}, \cdots, k_{1}, \cdots\right\}, T_{i}$ is a 6DOF rigid-body transform, and $z_{i}$ contains the $\mathrm{x}, \mathrm{y}$ pixel locations of the centers of every AprilTag in image $i$. In practice, we assume the joint distribution in Eqn. 9 can be approximated as multivariate Gaussian:

$$
\begin{aligned}
& N(x, \Sigma)=\left(\left[\begin{array}{c}
m \\
T_{0} \\
\vdots \\
T_{n}
\end{array}\right],\left[\begin{array}{cccc}
\Sigma_{m, m} & \Sigma_{m, T_{0}} & \cdots & \Sigma_{m, T_{n}} \\
\Sigma_{T_{0}, m} & \ddots & & \vdots \\
\vdots & & \ddots & \\
\Sigma_{T_{n}, m} & \cdots & & \Sigma_{T_{n}, T_{n}}
\end{array}\right]\right)
\end{aligned}
$$

This allows the marginal $P\left(m \mid z_{0}, \cdots\right)=N\left(m, \Sigma_{m, m}\right)$ to be computed trivially by dropping the other rows and columns of the covariance matrix. Computation of $\Sigma$ requires inverting the sparse $\operatorname{dim}(x)$ by $\operatorname{dim}(x)$ information matrix $\mathcal{I}$, which is derived from the observed target positions (similar to Eqn 1):

$$
\mathcal{I}=J^{T} \Sigma_{z}^{-1} J
$$

where each row of $J$ is the linearized projection equation describing how a point on the target projects into the image, given the model parameters $m$ and target position $T_{i}$. Crucially, this process depends on an estimate for the detector accuracy in pixels, $\sigma_{z}$, which must be known in advance. For AprilTag, we have empirically found the accuracy to be relatively constant across lenses with image width as a satisfactory predictor. Proper focus of the lens is assumed.

$$
\sigma_{z}=7 \times 10^{-5} \times \text { width }
$$

Detector accuracy was fit independently for a number of camera configurations (see Figure 8) using 60+ image calibration datasets for each. The resulting accuracies were then used to compute the linear model in Eqn. 12.

\section{IMPLEMENTATION}

AprilCal is implemented in Java and runs at 25 FPS with $640 \times 480$ images on a quad-core Intel i7-3740QM @ $2.7 \mathrm{GHz}$. Using a mosaic of AprilTags as our calibration target allows us to automatically detect the target at video rates, with processing time typically dominated by AprilTag 
detection. We have found that rigid mounting at an office supply store is inexpensive and yields a target durable enough for many uses. In addition, we can detect and recognize individual tags without observing the entire target. This makes it possible to add constraints in the corners of images, even for highly distorted lenses. In the multi-camera case, this allows calibration of cameras with adjacent, but nonoverlapping, fields of view.

In addition to target detection, our implementation requires significant CPU when determining the next suggestion. In our implementation, we score a fixed set of about 60 target positions regularly distributed throughout the field of view. This process depends on incorporating hypothetical observations into the calibration optimization framework, and then estimating the marginal distribution over the model parameters. As more images are acquired, and the size of the joint distribution grows, this can take up to 1 or 2 seconds. However, this scoring process only occurs a small number of times: once after each suggestion has been achieved by the user.

The AprilCal user interface is designed primarily to allow the user to correctly match the target positions suggested by the system. As shown in Figure 1, we use a set of unique colors to show how the desired target position (hollow rectangles) should be matched by the live detections (solid rectangles). The UI also automatically offers basic advice to the user via textual prompts about how to move the target to match the desired pose. When the calibration is deemed complete, the user is then automatically presented with the rectified video stream. This allows the user to qualitatively verify that the calibration is accurate, primarily by checking for straightness of projected lines.

\section{HUMAN TRIALS}

We conducted a series tests with human subjects ${ }^{2}$ to measure the effectiveness of AprilCal and to compare it to the widely used OpenCV method. Our user population consisted of undergraduate students at the University of Michigan. Only 3 of the 16 subjects reported any previous experience with camera calibration.

Our experiment protocol was as follows: each participant was asked to calibrate the same camera and mediumdistortion lens with two different methods (see Figure 3). We used a Point Grey Chameleon CMLN-13S2M-CS in $648 \times 482$ 8-bit grayscale mode with a $2.8 \mathrm{~mm}$ Tamron lens (Model 13FM28IR). This lens has a medium amount of distortion - significant enough that several Taylor series terms are required to model it, but still with a moderate field of view (only $93^{\circ}$ horizontal FOV).

The two methods we evaluated were OpenCV's calibration using automatic checkerboard detection and AprilCal using a mosaic of AprilTags. Participants were given a set of printed instructions. If they asked questions to the experimenter, they were given comprehension-level clarification on the

\footnotetext{
${ }^{2}$ Our study was reviewed by the University of Michigan Humanities Institutional Review Board and designated "exempt" with oversight number HUM00066852.
}

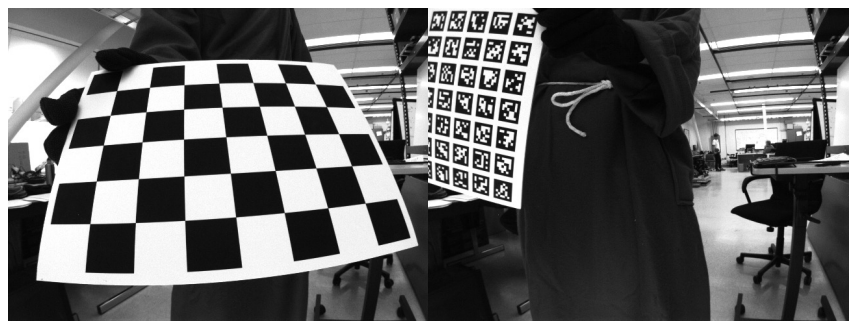

(a)

(b)

Fig. 3: Example images taken by the participants for both Method A (Open CV) and Method B (AprilCal).

instructions or advised to re-read the instructions. Participants then followed a checklist to first collect four samples using OpenCV, followed by four samples using AprilCal. Additionally, participants watched a video demonstrating calibration with each method. In Method A (OpenCV), participants interacted with a GUI showing live detections of the chessboard, using the "space" key on a wireless keyboard to capture a frame. In Method B (ApriCal), the frames are automatically taken when the participants move the targets close enough to the suggested pose.

In contrast to AprilCal, which provides detailed guidance throughout the calibration, OpenCV's calibrate.cpp provides no in-application suggestions. Therefore, we designed a set of instructions for calibrating with their software. Our goal was primarily to emulate the experience of a firsttime user who downloads this software from the Internet. Therefore, we provided users with some example pictures from the MATLAB Toolbox web page. The best written instructions we found were on the ROS tutorial for monocular camera calibration [18], which we also included. These are:

- checkerboard on the camera's left, right top and bottom of field of view

- checkerboard at various sizes

- close (fill the whole view)

- far (fill 1/5 of the view)

- checkerboard tilted to the left, right top and bottom

After reading these instructions, participants were then instructed to take 10-16 images in each of the 4 trials (on the same lens).

\section{Evaluation}

We evaluated AprilCal on several fronts. First, we report the results from our human subjects study to demonstrate the robustness of our approach to user error. Then, we demonstrate that our novel Max ERE metric is a good proxy for testing error. Finally, we demonstrate that AprilCal can be successfully used on a variety of lenses.

A note on evaluation of calibration quality: in all of our evaluations, we use testing error to indicate calibration quality. Each testing set is a collection of $60+$ images from all over the field of view, including the corners of the images and at various scales. Because we do not have ground truth positions for the targets in the testing set, the error we 


\begin{tabular}{lllr}
\hline \multirow{2}{*}{ Dataset } & \multirow{2}{*}{ Lens Model } & \multicolumn{2}{c}{ Reprojection Error } \\
\cline { 3 - 4 } & & Mean & Max \\
\hline OpenCV & Radial, 3 distortion terms & 0.728 & 38.646 \\
AprilCal & Radial, 3 distortion terms & 0.229 & 1.651 \\
AprilCal & Angular, 4 distortion terms & 0.203 & 1.444 \\
\hline
\end{tabular}

TABLE I: Mean and max testing errors averaged over all human subject trials in comparison to a 65 image reference dataset for the same lens. Results are significant with $p<$ $6.3 \times 10^{-7}$ for both mean and max errors and all pairs of rows.

report is after we optimize the target extrinsics to best fit the fixed model parameters for a given calibration. While this in general results in lower reprojection errors, it ensures that all models are fairly evaluated and still allows discrimination between good and bad calibrations.

Furthermore, Mean Reprojection Error (MRE) is typically reported as a summary of calibration quality, as it is simple to understand and robust to detector error. However, it can also often mask systematic errors in the underlying calibration. Therefore, we also report Max Reprojection Error on the test set - this ensures that calibrations are evaluated by their performance everywhere in the image.

\section{A. Novice Calibrators using AprilCal and OpenCV}

Our study results show that novices do a significantly better job calibrating when using AprilCal than when using OpenCV $\left(p<6.3 \times 10^{-7}\right)$. For example, with testing set errors averaged over all participants, the testing MRE using OpenCV is approximately three times that when using AprilCal (see Table I). The disparity is even greater when considering the Max Reprojection Errors - OpenCV averages 38 pixels (6\% of the image), whereas AprilCal averages a much lower 1.6 pixels for the same model. Interestingly, no OpenCV calibration yielded a max reprojection error better than the worst max reprojection error from AprilCal (2.02 pixels). This may be because the sorts of images that novice users capture, even when attempting to follow the ROS instructions, don't constrain the whole lens well. With the target suggestions provided by AprilCal, even new users of camera calibration software can produce calibrations with very low worst-case reprojection errors. The error histograms for both populations is shown in Figure 4.

The human study results can also help us understand where in the image the calibrations disagree. Figure 6 depicts the expected error between the human trials and a 65 image reference calibration. From the images shown, it is clear that the OpenCV calibrations fail to capture the lens model in the image corners. This can be explained by both the need to observe the whole calibration target in OpenCV and the difficulty for users to predict where constraints are needed.

In addition to showing that AprilCal calibrations are more accurate, the user study results also show that calibrations with AprilCal are more consistent. Figure 5 depicts the distribution of focal lengths and focal centers for both AprilCal and OpenCV. While both distributions have similar means,
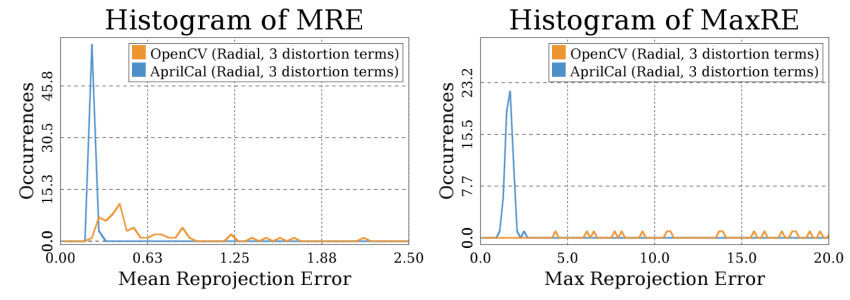

Fig. 4: Mean and Max Reprojection Errors (on a 65image test set) for calibrations produced using AprilCal and OpenCV. Users produced significantly more reliable calibrations using the proposed method $\left(p<6.3 \times 10^{-7}\right)$. For OpenCV, 3 MREs and 46 MaxREs not visible within plot extents.

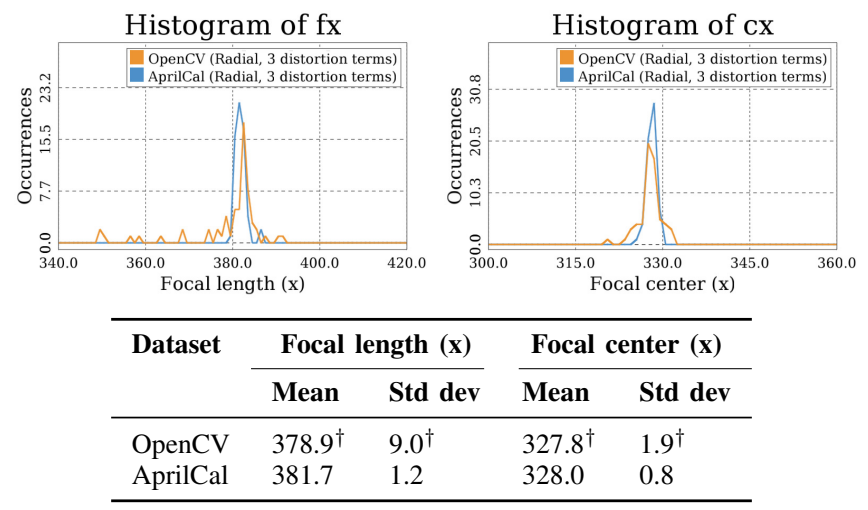

Fig. 5: Distribution of focal lengths and focal centers for all trials in the human study. While the mean parameter values from calibrations with AprilCal and OpenCV are similar, the standard deviations for the OpenCV calibrations are much higher. ${ }^{\dagger}$ One OpenCV outlier that would have further skewed the calculations was omitted from the calculations and is not visible within the plot extents. Best viewed in color.

the AprilCal standard deviations are $7.5 \times$ smaller for focal lengths and $2.37 \times$ smaller for focal centers.

\section{B. Evaluating the Max ERE metric}

We designed the Max ERE to be a good measure of calibration quality. Specifically, we want users to specify the accuracy they need for their application (e.g. $<1$ px), and if the Max ERE falls below that threshold, then the calibration can confidently be said to be that accurate. To validate these claims, we computed several variants of testing error over a large number of AprilCal trials. After each image is added, we evaluate the performance using Max ERE, as well as on an independent testing set using Max (100th percentile), 99.5th percentile, and mean reprojection errors. As can be seen in Figure 7, our sampled-MRE metric corresponds closely to the highest percentiles of testing error. This shows empirical evidence that our metric is effective.

\section{Accuracy of AprilCal on a Variety of Lenses}

In addition to performing reliably for a wide range of users, AprilCal also produces accurate calibrations for a 

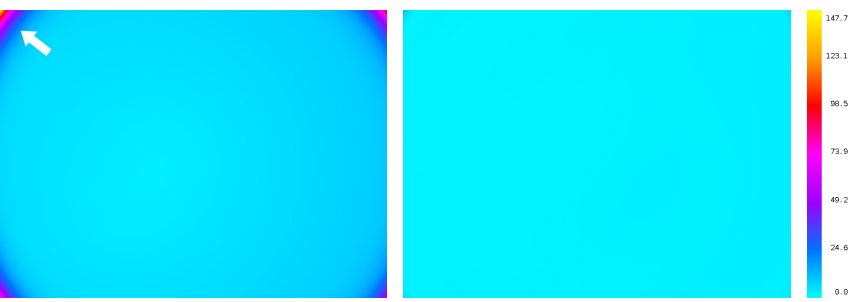

(a) OpenCV (Radial, 3 dist. terms) (b) AprilCal (Radial, 3 dist. terms)

\section{Cross section}

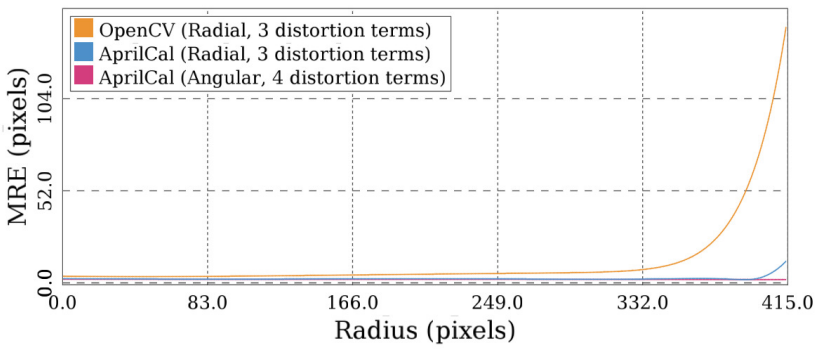

(d) Cross section from the focal center to the furthest corner in the image

Fig. 6: Heatmaps and cross section depicting per-pixel mean reprojection error between test subject calibrations and a 65 image testing set. Reprojection errors were calculated by projecting a point for every pixel in the reference calibration through all test subject calibrations, then computing the pixel distance. The reference calibration used an angular polynomial model with 4 terms, as it had the lowest mean and max training errors. AprilCal calibrations show low error in all parts of the image, while OpenCV calibrations have very high error in the corners (see arrow).

number of camera and lens configurations. Each lens was calibrated multiple times by one of the authors using the guidance provided by AprilCal (typically requiring 6-8 images in total). A separate $60+$ image testing set was collected to evaluate the accuracy for each configuration. To fairly compare results from different lenses, we compute each lens' testing error against a reference calibration computed from the corresponding test set. This eliminates the effects of detector error on testing error, which varies for different images sizes (See Eqn 12). For each target point detected in the testing set, we project through both the reference calibration and the calibration using AprilCal, and compute the Mean, 99.5 $5^{\text {th }}$ percentile and Max Reprojections Errors. Figure 8 shows the testing error for six configurations that our lab uses for various robotics applications, including stereo odometry, object detection and overhead ground truth. In each case, the testing MRE is significantly below one pixel. Example images from each configuration are shown in Figure 9.

\section{SUMMARY}

AprilCal is an interactive calibration tool that provides live feedback on the state of the calibration and produces tightly-distributed calibration parameters even when used by

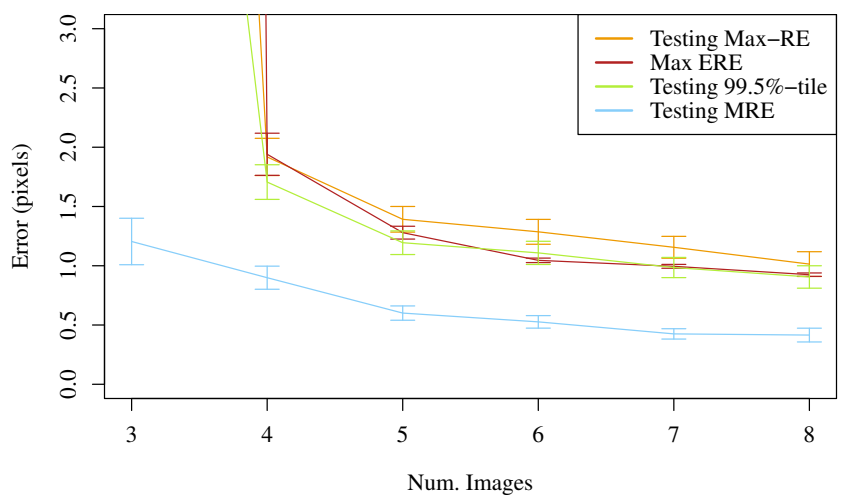

Fig. 7: Our novel Max Expected Reprojection Error (Max ERE) metric (red) correlates highly with the 99.5 th percentile of reprojection errors on an independently captured test set. The Max ERE allows us to automatically compute a rigorous accuracy score for a partial calibration without needing an exhaustive test set. Error bars reflect std. error of the mean.

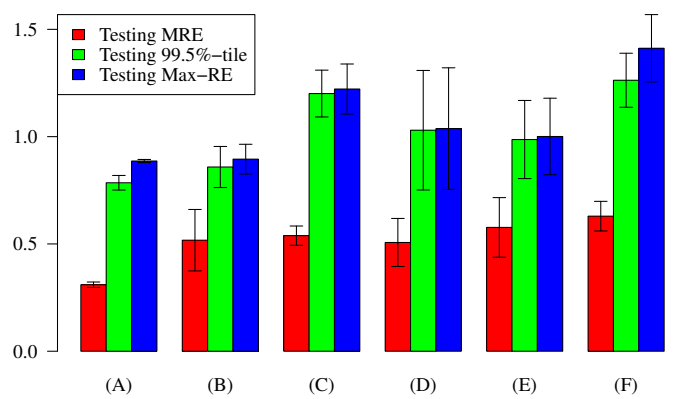

\begin{tabular}{llrrl}
\hline & Lens & DFOV & Resolution & Format \\
\cline { 2 - 5 } (a) & Fujinon YV2.2x1.4A-2 & $143^{\circ}$ & $648 \times 482$ & Gray \\
(b) & Tamron 13FM22IR & $146^{\circ}$ & $648 \times 482$ & Gray \\
(c) & Tamron 13FM28IR & $114^{\circ}$ & $648 \times 482$ & Gray \\
(d) & Boowon BW38B & $83^{\circ}$ & $752 \times 480$ & Color \\
(e) & Boowon BW3M30B & $121^{\circ}$ & $648 \times 482$ & Gray \\
(f) & Boowon BW3M30B & $121^{\circ}$ & $1296 \times 964$ & Color \\
\hline
\end{tabular}

Fig. 8: Testing error for a variety of camera configurations using AprilCal. The Diagonal Field Of View (DFOV) was estimated from the testing sets for each configuration. Error bars reflect std. error of the mean.

novices. We have leveraged a novel calibration quality metric (Max ERE) to automatically determine whether a calibration is sufficiently accurate, without requiring a user to collect a rigorous testing set. We conducted a 16-person human subjects study to show that even novice users can produce consistent, quality calibrations using such a system.

We have evaluated AprilCal in a variety of ways, and demonstrated that it is a suitable replacement for the currently available calibration toolkits, which use a batch calibration process and training error as a quality metric. Our desire is to make accurate camera calibration available to a wider audience who can use the resulting model parameters confidently in a range of applications. 


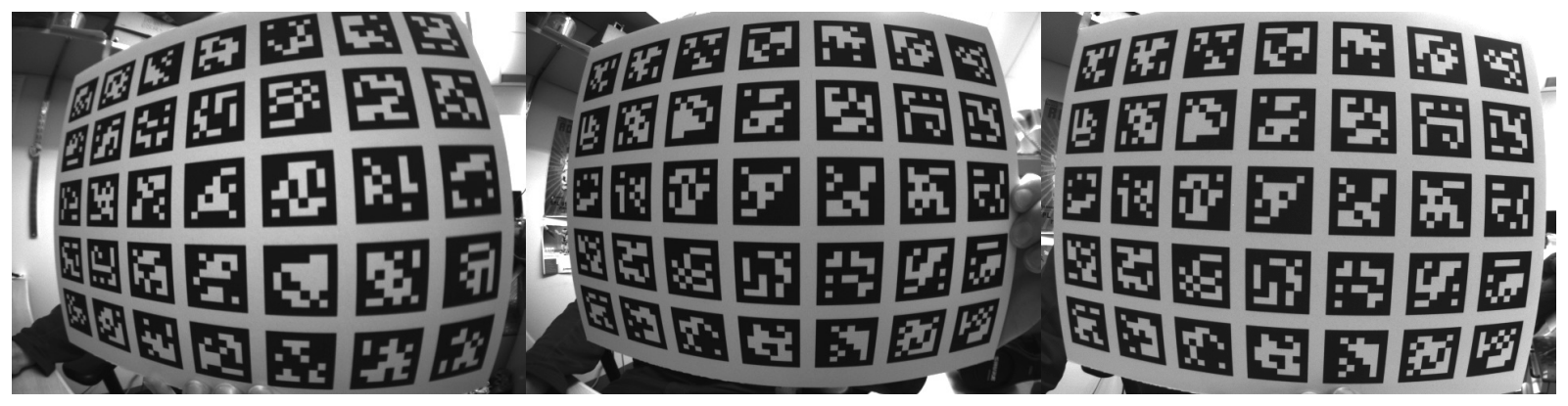

(a) Fujinon YV2.2x1.4A-2 $(648 \times 482)$

(b) Tamron 13FM22IR $(648 \times 482)$

(c) Tamron 13FM28IR $(648 \times 482)$

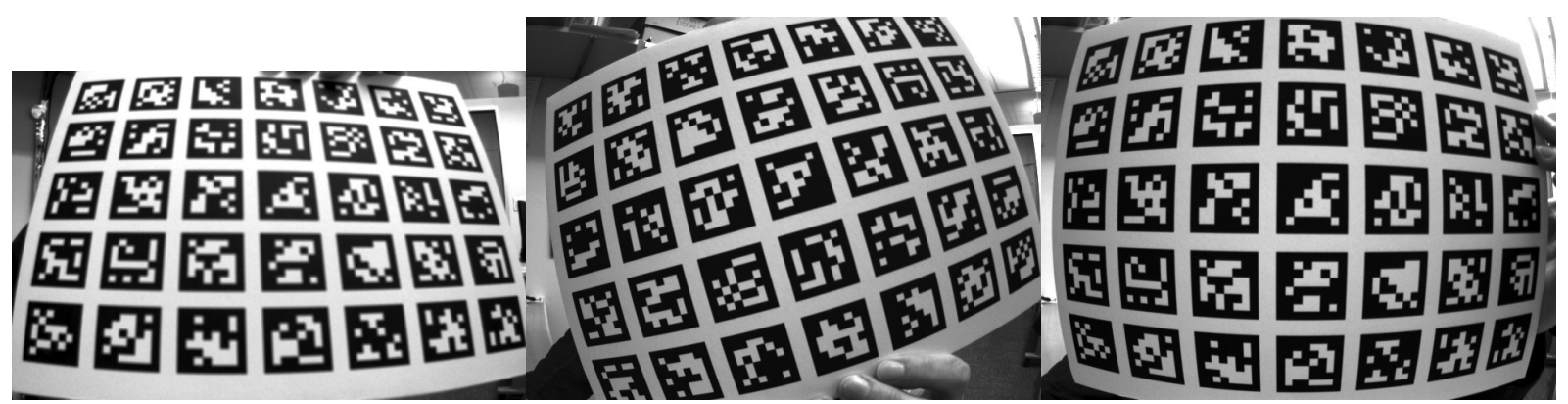

(d) Boowon BW38B $(752 \times 480)$

(e) Boowon BW3M30B $(648 \times 482)$

(f) Boowon BW3M30B $(1296 \times 964)$

Fig. 9: Example images for each of the configurations evaluated in Figure 8. These lenses are representative of those known to work with AprilCal. Image contrast adjusted for clarity.

\section{ACKNOWLEDGMENTS}

This work was funded by U.S. DoD Grant FA2386-11-14024.

\section{REFERENCES}

[1] J. Barreto and K. Daniilidis. Wide area multiple camera calibration and estimation of radial distortion. In Omnivis-2004, ECCV-2004 workshop, 2004.

[2] J. Barreto, J. Roquette, P. Sturm, F. Fonseca, et al. Automatic camera calibration applied to medical endoscopy. In 20th British Machine Vision Conference (BMVC'09), 2009.

[3] J.-Y. Bouguet. Camera calibration toolbox for MATLAB, July 2010.

[4] G. Bradski. The OpenCV Library. Dr. Dobb's Journal of Software Tools, 2000

[5] F. Dellaert and M. Kaess. Square root SAM: Simultaneous localization and mapping via square root information smoothing. International Journal of Robotics Research, 25(12):1181-1203, December 2006.

[6] F. Devernay and O. Faugeras. Straight lines have to be straight. Machine Vision and Applications, 13(1):14-24, 2001.

[7] D. Forsyth and J. Ponce. Computer Vision: A Modern Approach. Prentice Hall Professional Technical Reference, 2002.

[8] A. Geiger, J. Ziegler, and C. Stiller. StereoScan: Dense 3d reconstruction in real-time. In IEEE Intelligent Vehicles Symposium, BadenBaden, Germany, June 2011.

[9] R. Hartley and A. Zisserman. Multiple View Geometry in Computer Vision. Cambridge University Press, second edition, 2004.

[10] J. Kannala and S. S. Brandt. A generic camera model and calibration method for conventional, wide-angle, and fish-eye lenses. Pattern Analysis and Machine Intelligence, IEEE Transactions on, 28(8):1335-1340, 2006.

[11] K. Konolige. Sparse sparse bundle adjustment. In British Machine Vision Conference, Aberystwyth, Wales, August 2010.
[12] R. Kummerle, G. Grisetti, H. Strasdat, K. Konolige, and W. Burgard. g2o: A general framework for graph optimization. In ICRA, Shanghai, May 2011.

[13] M. Li. Camera calibration of a head-eye system for active vision. Computer VisionECCV'94, pages 541-554, 1994

[14] C. Mei, G. Sibley, M. Cummins, P. Newman, and I. Reid. RSLAM A system for large-scale mapping in constant-time using stereo. International Journal of Computer Vision, pages 1-17, 2010.

[15] R. A. Newcombe and A. J. Davison. Live dense reconstruction with a single moving camera. In Computer Vision and Pattern Recognition (CVPR), 2010 IEEE Conference on, pages 1498-1505. IEEE, 2010.

[16] E. Olson. AprilTag: A robust and flexible visual fiducial system. In Proceedings of the IEEE International Conference on Robotics and Automation (ICRA), May 2011.

[17] V. Pradeep, K. Konolige, and E. Berger. Calibrating a multi-arm multi-sensor robot: A bundle adjustment approach. In International Symposium on Experimental Robotics (ISER), New Delhi, India, 12/2010 2010.

[18] M. Quigley, B. Gerkey, K. Conley, J. Faust, T. Foote, J. Leibs, E. Berger, R. Wheeler, and A. Ng. Ros: an open-source robot operating system. In ICRA workshop on open source software, volume 3, 2009

[19] P. Ranganathan and E. Olson. Gaussian process for lens distortion modeling. In Proceedings of the IEEE/RSJ International Conference on Intelligent Robots and Systems (IROS), October 2012.

[20] J. Strom, A. Richardson, and E. Olson. Graph-based segmentation for colored 3D laser point clouds. In Proceedings of the IEEE/RSJ International Conference on Intelligent Robots and Systems (IROS), October 2010.

[21] T. Svoboda. A software for complete calibration of multicamera systems. In Electronic Imaging 2005, pages 115-128. International Society for Optics and Photonics, 2005.

[22] Z. Zhang. A flexible new technique for camera calibration IEEE Transactions on Pattern Analysis and Machine Intelligence, 22(11):1330-1334, 2000. 